PROCEEDINGS OF THE

AMERICAN MATHEMATICAL SOCIETY

Volume 132, Number 2, Pages 499-501

S 0002-9939(03)07142-9

Article electronically published on June 23, 2003

\title{
REMARK ON THE STRONG UNIQUE CONTINUATION PROPERTY FOR PARABOLIC OPERATORS
}

\author{
GIOVANNI ALESSANDRINI AND SERGIO VESSELLA \\ (Communicated by Andreas Seeger)
}

\begin{abstract}
We consider solutions $u=u(x, t)$, in a neighbourhood of $(x, t)=$ $(0,0)$, to a parabolic differential equation with variable coefficients depending on space and time variables. We assume that the coefficients in the principal part are Lipschitz continuous and that those in the lower order terms are bounded. We prove that, if $u(\cdot, 0)$ vanishes of infinite order at $x=0$, then $u(\cdot, 0) \equiv 0$.
\end{abstract}

Consider, in the ball $B=B_{1}(0,0)$ of $\mathbb{R}^{n+1}$, the parabolic differential operator

$$
L(u)=u_{t}-\sum_{i, j=1}^{n} a_{i j} u_{x_{i} x_{j}}
$$

here $x \in \mathbb{R}^{n}, t \in \mathbb{R}$. Let us assume that $\left\{a_{i j}(x, t)\right\}_{i, j=1}^{n}$ is a symmetric matrix satisfying, for some $\lambda, 0<\lambda \leq 1$, the uniform ellipticity condition

$$
\lambda|\xi|^{2} \leq \sum_{i, j=1}^{n} a_{i j}(x, t) \xi_{i} \xi_{j} \leq \lambda^{-1}|\xi|^{2}, \text { for every }(x, t) \in B, \xi \in \mathbb{R}^{n} .
$$

Let us also assume that the following Hölder condition is satisfied for some $\alpha$, $0<\alpha \leq 1$, and some $M>0$ :

$$
\begin{aligned}
\sum_{i, j=1}^{n}\left|a_{i j}(x, t)-a_{i j}(y, s)\right| & \leq M\left(|x-y|^{2}+|t-s|\right)^{\frac{\alpha}{2}}, \\
& \text { for every }(x, t),(y, s) \in B .
\end{aligned}
$$

Recently, it has been proven by Escauriaza and Fernandez (3]) that if $\alpha=1$, then the following strong unique continuation property holds.

Property 1 (SUCP). For every $u$ that satisfies the differential inequality

$$
|L(u)| \leq C\left(\left|D_{x} u\right|+|u|\right) \text { in } B,
$$

for some $C>0$, if we have

$$
u(x, t)=O\left(\left(|x|^{2}+|t|\right)^{\frac{k}{2}}\right) \text { for } t \leq 0 \text { and for every } k \in \mathbb{N},
$$

Received by the editors October 15, 2002.

2000 Mathematics Subject Classification. Primary 35B05, 35K99; Secondary 35R25.

Key words and phrases. Strong unique continuation, parabolic equations.

The authors acknowledge partial support from M.U.R.S.T. grant no. MM01111258. 
then we have

$$
u(\cdot, 0) \equiv 0 \text {. }
$$

It seems that a natural refinement of the above result would be the following.

Property 2 (Spacelike-SUCP). For every $u$ which satisfies the differential inequality (44)) for some $C>0$, if we have

$$
u(x, 0)=O\left(|x|^{k}\right) \text { for every } k \in \mathbb{N},
$$

then we have ([6) ).

Here we give a short proof of the fact that if $\alpha=1$, then also the Spacelike-SUCP holds. It is worth mentioning that such property was proven by Fang-Hua Lin [6] for solutions $u$ of parabolic equations with time independent coefficients

$$
u_{t}-\sum_{i, j=1}^{n} a_{i j}(x) u_{x_{i} x_{j}}-\sum_{i=1}^{n} b_{i}(x) u_{x_{i}}-c(x) u=0,
$$

when the coefficients $a_{i j}$ satisfy (2) and (3) with $\alpha=1$ and the coefficients $b_{i}, c$ are bounded. Therefore, the main novelty here stands on the fact that coefficients are allowed to depend on all space and time variables. More precisely, we prove.

Theorem 1. Let $L$ be as in (11) and let us assume (2) and (3). If $L$ satisfies the $S U C P$, then it also satisfies the Spacelike-SUCP.

The proof will be based on the following result on the local behaviour of solutions to parabolic equations which was obtained by the authors in [1], Theorem 1.1. In the sequel, we shall say that a polynomial $P(x, t)$ is $p$-homogeneous of degree $m \in \mathbb{N}$ if $P\left(\lambda x, \lambda^{2} t\right)=\lambda^{m} P(x, t)$ for every $\lambda>0,(x, t) \in \mathbb{R}^{n+1}$. We also set

$$
\begin{gathered}
E_{0}(u)=\sum_{i, j=1}^{n} a_{i j}(0,0) u_{x_{i} x_{j}}, \\
L_{0}(u)=u_{t}-E_{0}(u) .
\end{gathered}
$$

Theorem 2. Let $L$ be as in (1) and let us assume (2) and (3). For every u which satisfies the differential inequality (4) either one of the following two facts occurs:

(I) property (5) holds,

(II) there exist $m \in \mathbb{N}$ and a nonzero p-homogeneous polynomial $P_{m}(x, t)$ of degree $m$ such that

$$
u(x, t)=P_{m}(x, t)+o\left(\left(|x|^{2}+|t|\right)^{m / 2}\right)
$$

and

$$
L_{0}\left(P_{m}\right)=0 .
$$

The above theorem is a generalization to parabolic equations of a well-known result of Bers [2] on the local asymptotic behaviour of solutions of elliptic equations. More recent developments along this line can be found in [5].

Proof of Theorem 1. Let $u$ be a solution of (4) which satisfies (7) and suppose that (10) holds for some $m \in \mathbb{N}$ and some $p$-homogeneous polynomial $P_{m}(x, t)$ of degree $m$ which satisfies (11). Therefore,

$$
P_{m}(x, 0)=o\left(|x|^{m}\right) .
$$


and, by the homogeneity of degree $m$ in the $x$-variables, $P_{m}(\cdot, 0) \equiv 0$. Therefore, by (11),

$$
D_{x}^{\beta} D_{t}^{k} P_{m}(0,0)=D_{x}^{\beta} E_{0}^{k}\left(P_{m}\right)(0,0)=0
$$

for every multi-index $\beta$ and for every $k \in \mathbb{N}$, hence $P_{m} \equiv 0$. Consequently, by Theorem 2, case (I) holds and, by the SUCP, (6) follows.

\section{ACKNOWLEDGEMENT}

We wish to thank Luis Escauriaza for stimulating conversations which led us to consider the topic of the present note, and for having shared with us the results of his ongoing joint work with F. J. Fernandez. In fact, while this note was in preparation, he informed us that F. J. Fernandez ([4]) has independently obtained another proof of Property 2 when $\alpha=1$, by different methods.

\section{REFERENCES}

1. Alessandrini, G. and Vessella, S., Local behaviour of solutions to parabolic equations, Comm. Partial Differential Equations, 13 (9), (1988), 1041-1058. MR 89h:35140

2. Bers, L. Local behavior of solutions of general linear elliptic equations, Comm. Pure Appl. Math. 8 (1955), 473-496. MR 17:743a

3. Escauriaza, L. and Fernandez, F. J., Unique continuation for parabolic operators, to appear in Ark. Mat.

4. Fernandez, F.J., Unique continuation for parabolic operators II, preprint.

5. Han, Qing, On the Schauder estimates of solutions to parabolic equations, Ann. Scuola Norm. Sup. Pisa Cl. Sci. (4) 27(1) (1998), 1-26. MR 2000a:35094

6. Lin, Fang-Hua, A uniqueness theorem for parabolic equations. Comm. Pure Appl. Math. 43(1) (1990), 127-136. MR 90j:35106

Dipartimento di Scienze Matematiche, Universitá degli Studi di Trieste, Via A. VALeRIO 12/1, 34127 Trieste, ItAly

E-mail address: alessang@univ.trieste.it

Dimad, Universitá degli Studi di Firenze, Via C. Lombroso 6/17, 50134 Florence, ITALY

E-mail address: vessella@dmd.unifi.it 